Rev. Elev. Méd. vét. Pays trop., 1978, 31 (3) : 329-340.

\title{
Contribution à l'étude de la répartition des glossines dans le nord de la Côte-d'Ivoire
}

\author{
par Y. TAZE $\left({ }^{*}\right)$, D. CUISANCE $\left({ }^{*}\right)$, M. ClAIR $\left(^{*}\right)$ H. POLITZAR $\left(^{*}\right)$
}

\begin{abstract}
RÉSUME
Six espèces ont été recensées: G. p. gambiensis, G. tachinoides, G. $m$. submorsitans, $G$. longipalpis, $G$. fusca fusca, $G$. fusca congolensis.

Bien que ces enquêtes ponctuelles ne donnent qu'une idée fragmentaire des répartitions des espèces et de Ieurs densités, elles ont permis de noter l'existence de foyers non signalés dans les cartes de répartition antérieures, notamment pour $G$. tachinoides et $G, m$. submorsitans.
\end{abstract}

\section{INTRODUCTION}

Aucune carte de répartition des glossines sur l'ensemble de la Côte-d'Ivoire n'a jusqu'ici été publiée. Il existe seulement plusieurs cartes à petite échelle, intéressant soit l'Afrique Occidentale d'expression française, soit l'ensemble de l'Afrique au sud du Sahara.

ROUBAUD, en 1920, présente la première carte de répartition des glossines dans l'ancienne fédération, dressée à la suite des «Missions BOUET-ROUBAUD, 1906-1916 ".

En 1948-1949, VILAIN publie une carte au $1 / 3000000^{\mathrm{e}}$ sur la répartition des glossines en Afrique Occidentale francophone.

Ces données sont reprises en 1953 par POTTS qui établit une carte de répartition au $1 / 5000000^{\mathrm{e}}$, pour l'ensemble de l'Afrique.

A la suite de nouvelles prospections, RICKENBACH publie en 1961 une carte au $1 / 10000000^{\mathrm{e}}$, de l'Afrique Occidentale, qui mentionne la présence des glossines par degré carré.

Enfin en 1963 FORD, et en 1973 FORD et KATONDO présentent des cartes de répartition pour l'ensemble de l'Afrique, qui reprennent les données antérieures.

(*) C. R. T. A. : Centre I. E. M. V. T. de Recherche sur les Trypanosomiases Animales.
Ces cartes sont fort imprécises en raison de leurs trop petites échelles. Depuis leur établissement, des modifications apportées par l'homme à la végétation, et aux densités de gibier et de bétail ainsi que les variations climatiques de ces dernières années ont peut être entraîné des bouleversements dans les aires de répartition des diverses espèces de glossines.

Des enquêtes récentes ont été réalisées à la demande du gouvernement ivoirien, soit par l'O. C. C. G. E. $\left({ }^{* *}\right)$, dans les foyers de trypanosomiase humaine, soit par la W. I. P. $\left({ }^{* *}\right)$, lors de la mise en place de zones d'aménagement.

Dans le même temps et à la demande des autorités ivoiriennes, les entomologistes du C. R. T. A. ( $\left.{ }^{*}\right)$ ont effectué des enquêtes ponctuelles, de 1971 à 1977, destinées à évaluer la contrainte exercée par les glossines, dans des zones d'aménagement pastoral du nord de la Côte-d'Ivoire.

La présente publication rassemble, sous la forme d'une carte au $1 / 800000^{\mathrm{e}}$, les résultats collectés au cours de ces dernières enquêtes.

(**) O. C. C. G. E. : Organisation de Coordination et de Coopération pour la lutte contre les Grandes Endémies.

$(* * *)$ W. I. P. : Wirtschaft und Infrastruktur Plannungs. 


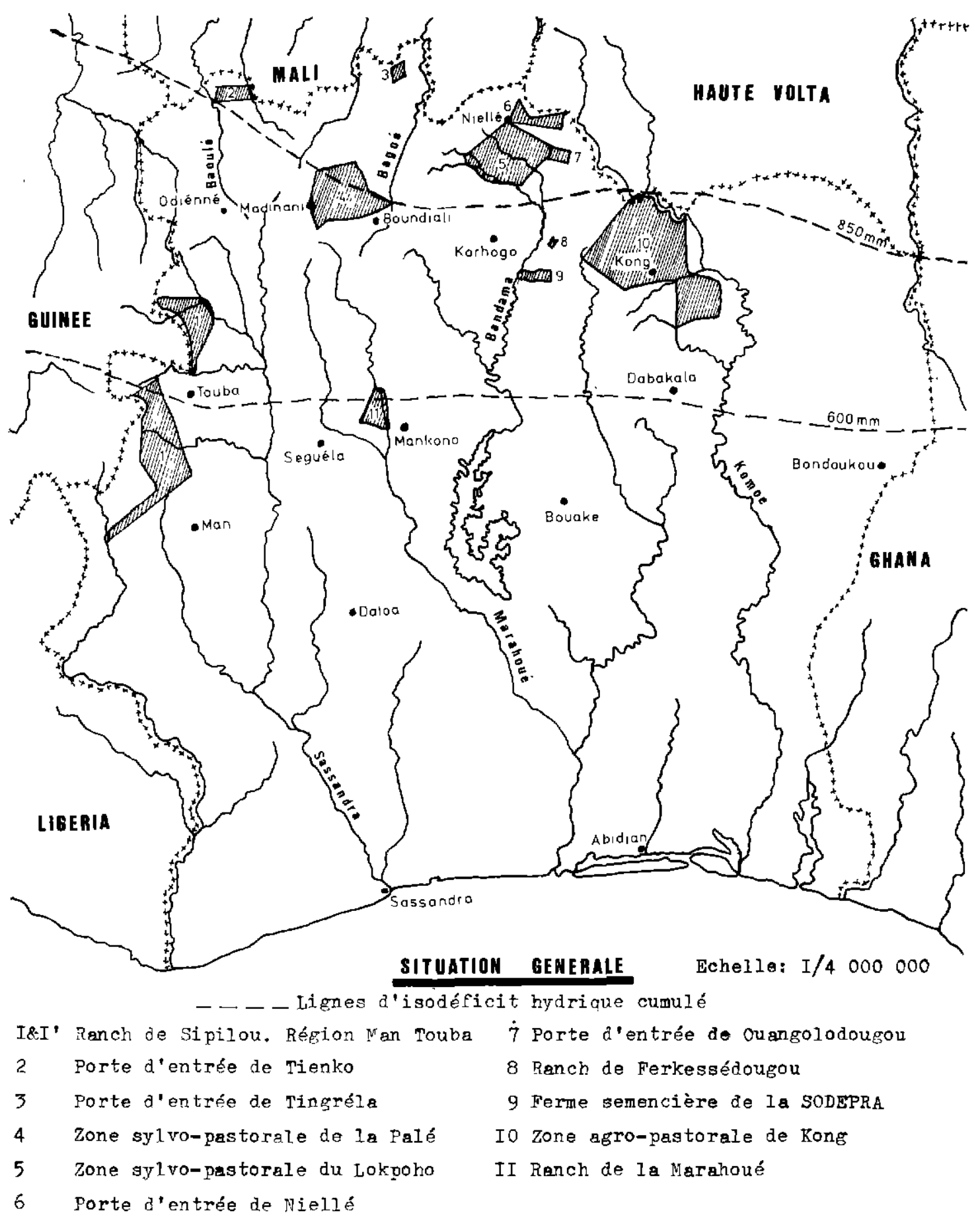

\section{ENQUÊTES EFFECTUÉES} PAR L'ÉQUIPE DU C. R. T. A.

II. 1. Milieu physique du nord de la Côted'Ivoìre

\section{Situation géographique}

La zone des savanes du nord de la Côted'Ivoire est limitée par la frontière avec le Mali et la Haute-Volta (environ $10^{\circ} 50^{\prime}$ de latitude nord), les frontières avec la Guinée (environ $8^{\circ}$ de longitude ouest) et le Ghana (environ $3^{\circ}$ de longitude est). Vers le sud, elle est limitée par la bordure nord de la zone forestière, qui suit approximativement le $8^{\circ}$ parallèle nord, mais s'infléchit au centre jusque dans la région de Toumodi en formant le «V Baoulé ». 


\section{Relief}

La dorsale guinéenne, bourrelet de hautes terres qui prolonge vers l'est le massif du FoutaDjalon, pénètre en Côte-d'Ivoire au nord-ouest. Elle se présente comme une succession de plateaux d'altitude moyenne de l'ordre de $400 \mathrm{~m}$ avec quelques reliefs dominants. $\mathrm{Ce}$ socle granitique s'abaisse tant au nord qu'au sud, selon une ligne NE-SW.

La région de Man et Touba se caractérise par une succession de collines et de chaînons culminants parfois à 1100 ou $1200 \mathrm{~m}$, donnant un relief très mamelonné.

La région de Tienko, Odiénné, Madinani et Boundiali est formée par le socle granitique à une altitude moyenne de 400 à $500 \mathrm{~m}$ avec des reliefs individuels (inselbergs granitiques) qui prennent une grande importance.

La région centrale et nord-est représente un glacis dont l'altitude décroît, en $350 \mathrm{~km}$, de 400 . $450 \mathrm{~m}$ dans la région de Boundiali à $300 \mathrm{~m}$ dans la vallée de la Komoë. Des plateaux rigides cuirassés, sur schistes ou granits, forment la majeure partie du paysage. Quelques reliefs isolés interrompent, par place, la monotonie des plateaux.

La région de Mankono et Séguéla est formée de plateaux s'abaissant modérément du nord vers le sud entre 400 et $300 \mathrm{~m}$. Elle fait transition entre la dorsale guinéenne proprement dite et les plateaux du nord. Sur ce socle granitique se sont développés entre Séguéla et Mankono des inselbergs cristallins typiques.

\section{Hydrographie}

Le réseau hydrographique du nord de la Côte-d'Ivoire se divise en 4 grands systèmes :

- le versant nigérien qui intéresse le nordouest : région de Tienko, Odiénné, Madinani et Boundiali, avec les 2 affluents importants que sont la Baoulé et la Bagoé ;

- le bassin du Sassandra qui draine les eaux de l'ouest : région de Man et Touba.

- le bassin du Bandama (Bandama blanc et Marahoué) qui draîne toute la région centrale ;

- le bassin de la Komoë, à l'est d'une ligne Ferkéssédougou-Dabakala.

Ce réseau hydrographique relativement dense présente, à l'exception des grands fleuves, un régime temporaire, les petits cours d'eau s'assé- chant une grande partie de l'année et ayant un régime torrentiel pendant la saison des pluies.

\section{Climat}

\section{Pluviométrie}

La moyenne annuelle des hauteurs de précipitations varie d'ouest en est de $1700 \mathrm{~m}$ (Man) à $1100 \mathrm{~mm}$ (Bondoukou). Ces précipitations se répartissent sur 6 à 8 mois de l'année.

\section{Températures}

La moyenne annuelle des températures varie du nord au sud de $27,5^{\circ} \mathrm{C}$ à $25^{\circ} \mathrm{C}$.

\section{Etat hygrométrique}

La moyenne annuelle de l'humidité relative varie du nord au sud de 63 p. 100 à 79 p. 100.

La ligne d'isodéficit hydrique cumulé $850 \mathrm{~mm}$, représentant la limite entre secteurs soudanien et sub-soudanien (1), traverse la zone d'ouest en est au nord d'Odiénné, Boundiali, Korhogo, Ferkéssédougou et Bouna.

La ligne d'isodéficit hydrique cumulé $600 \mathrm{~mm}$ représentant la limite entre secteurs sub-soudanien et guinéen (1), traverse la zone d'ouest en est au sud de Touba, au nord de Mankono, au sud de Dabakala et au nord de Bondoukou.

\section{Végétation}

Du nord-est au sud-ouest, se succèdent la savane arbustive et arborée, la savane boisée, la forêt claire et à l'extrême sud des îlots de forêt humide semi-décidue (région de Man).

Les cours d'eau permanents ou temporaires sont bordés par des galeries forestières importantes, généralement continues, moyennement larges et denses.

Des îlots forestiers ( «bois sacrés ») signalent fréquemment les villages.

En plus des cultures vivrières (mil, sorgho, patates douces et ignames) les cultures industrielles (riz, coton, canne à sucre) se développent de plus en plus.

\section{2. Localisation des zones prospectées (cf. carte générale)}

De 1971 à 1977, des enquêtes entomologiques ont été effectuées dans les zones d'aménagement suivantes. 


\section{Ranch de Sipilou}

Situé au nord-ouest de Man, ce ranch est à la limite des zones sub-soudanienne et guinéenne. Deux cours d'eau le bordent, le Bafing et son affluent le Méné. La population humaine est inexistante. Le bétail est composé de taurins N'Dama et une faune résiduelle de grands mammifères (bubales) cohabite avec les bovins.

\section{Région de Man, Touba, Borotou}

A l'extérieur du ranch de Sipilou, des points isolés ont été prospectés entre la route Borotou, Man et la frontière guinéenne.

\section{Ranch de la Marahoué}

Situé à la limite des zones sub-soudanienne et guinéenne, ce ranch a été créé pour assurer un élevage naisseur de taurins N'Dama. Il est limité à l'est par la Marahoué et comporte quelques îlots importants de forêt dense sèche. La population humaine en est absente. Quelques grandes antilopes (bubales) et des phacochères cohabitent avec les troupeaux.

\section{Porte d'entrée du bétail de Tienko}

Près de la frontière malienne, cette région, située en secteur soudanien, se trouve sur une route traditionnelle du bétail entrant en Côted'Ivoire. Elle intéresse le bassin versant de la Baoulé. La région est peu peuplée et seuls quelques troupeaux taurins y vivent en permanence. Une faune assez abondante, représentée essentiellement par des phacochères, guibs harnachés et cobs de Buffon, y est encore présente.

\section{Porte d'entrée du bétail de Tingréla}

Le périmètre retenu est très réduit et se trouve à mi-chemın entre la vallée de la Bagoé et celle de la Kobani. Les quelques marigots traversant cette zone sont tous à sec en saison sèche. Du bétail taurin est assez bien représenté près des villages et des troupeaux de zébus transhument dans cette région. Quelques peuhls sédentaires pratiquent le métissage de leurs animaux avec des taurins. Pas de faune sauvage, à l'exception de quelques phacochères, guibs harnachés et de varans.

Portes d'entrée du bétail de Niellé et Ouangolodougou et zone sylvo-pastorale du Lokpoho

Ces 3 zones jointives sont à cheval sur la limite des secteurs soudanien et sub-soudanien. Le nord est intéressé par le bassin versant de la
Léraba, elle-même affluent de la Komoë. Le sud est draîné par les affluents du Bandama. La population y est relativement importante et pratique des cultures vivrières. La culture du coton se développe. On trouve dans chaque village des troupeaux de taurins ou de métis taurins $\times$ zébus. Une assez forte population peuhl $y$ effectue de petites transhumances avec des troupeaux de zébus. La grande faune a pratiquement disparu, sauf dans l'ex-forêt classée du Badénou et des rives de la Léraba. Une petite faune mammalienne et reptilienne habite les galeries forestières.

\section{Zone d'aménagement sylvo-pastoral de la Palé}

Située entre Madinani et Boundiali, cette région est en bordure de la dorsale guinéenne. Son climat est typiquement sub-soudanien. Les eaux s'écoulent vers le nord par les affluents de la Bagoé. Constituée autour de l'ancienne forêt classée de la Palé, cette zone vide de population est destinée à la sédentarisation de troupeaux de zébus peuhl. Au centre de la zone, une faune de grands mammifères (buffles, hippotragues, bubales, cobs defassa, cobs de Buffon phacochères, lycaons, panthères, cynocéphales) est encore bien représentée.

\section{Ferme semencière de la SODEPRA}

A l'ouest de Badikaha, limitée par le Bandama et son affluent le Farakouo, cette ferme est destinée à la culture semencière de plantes fourragères. Le terrain est presque entièrement déboisé, à l'exception de quelques blocs de forêt claire sèche et des galeries forestières. Un petit troupeau de taurins N'Dama et Baoulé y est embouché sur Stylosanthes. Une faune résiduelle de grands mammifères profite de ces cultures.

\section{Zone agro-pastorale de Kong}

Il s'agit d'une vaste région limitée au nord par la Komoë et à l'est par le Parc National de la Komoë. Cette région située en secteur subsoudanien, à végétation formée d'une savane arbustive et arborée avec des zones de forêt claire, n'abrite qu'une faible population humaine. Quelques milliers de têtes de taurins vivent près des villages. La proximité du Parc National et de forêts classées, ainsi que l'existence de grandes zones de savane inhabitée expliquent qu'une faune sauvage relativement abondante peut y être observée. 


\section{CARTE $n \cdot 2$ - Nord Cotoute ${ }^{\text {Reny I I }}$}

REPARTITION DES GLOSSINES - Synthèse des enquêtes du C.R.TA. לn

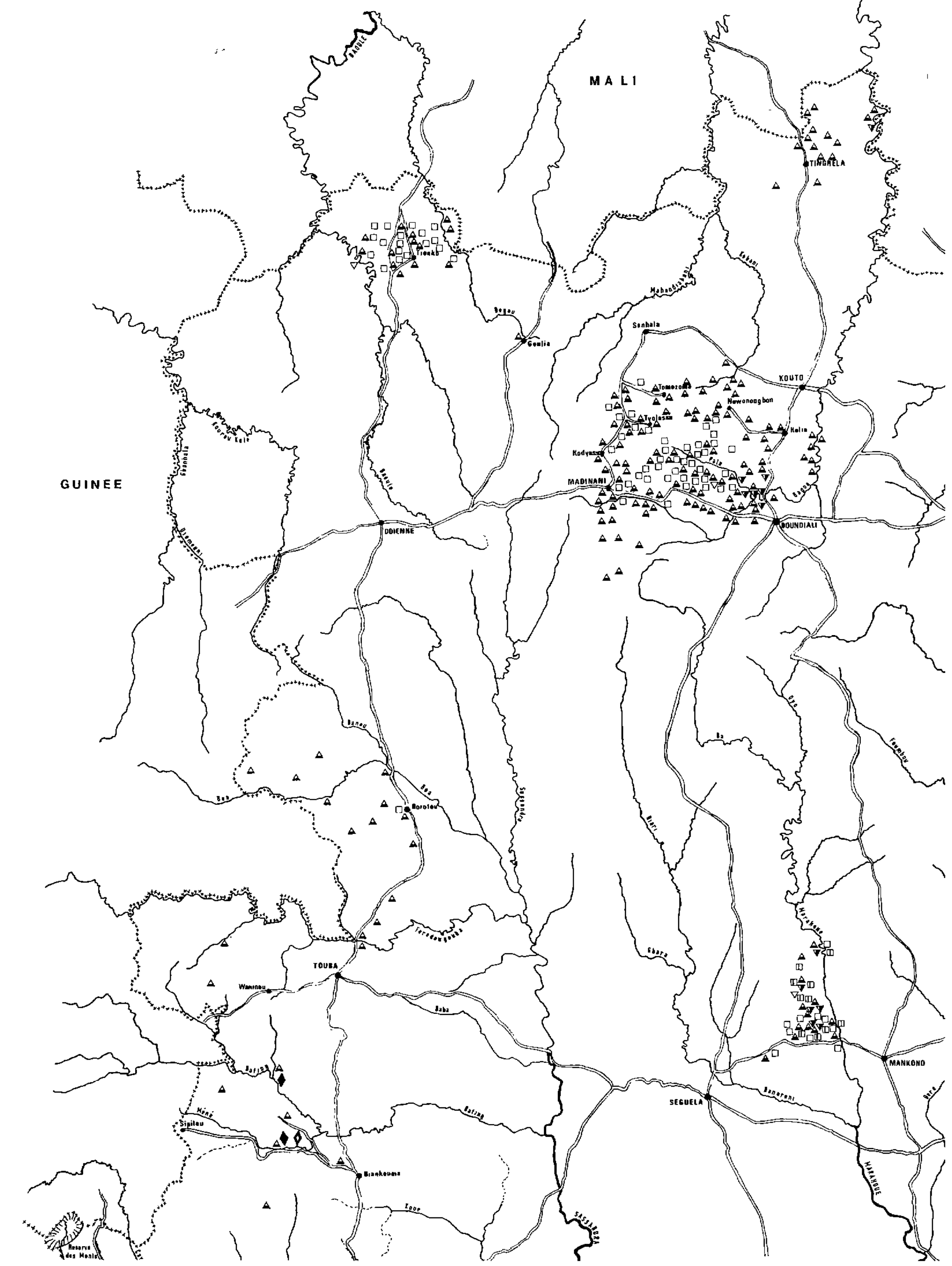




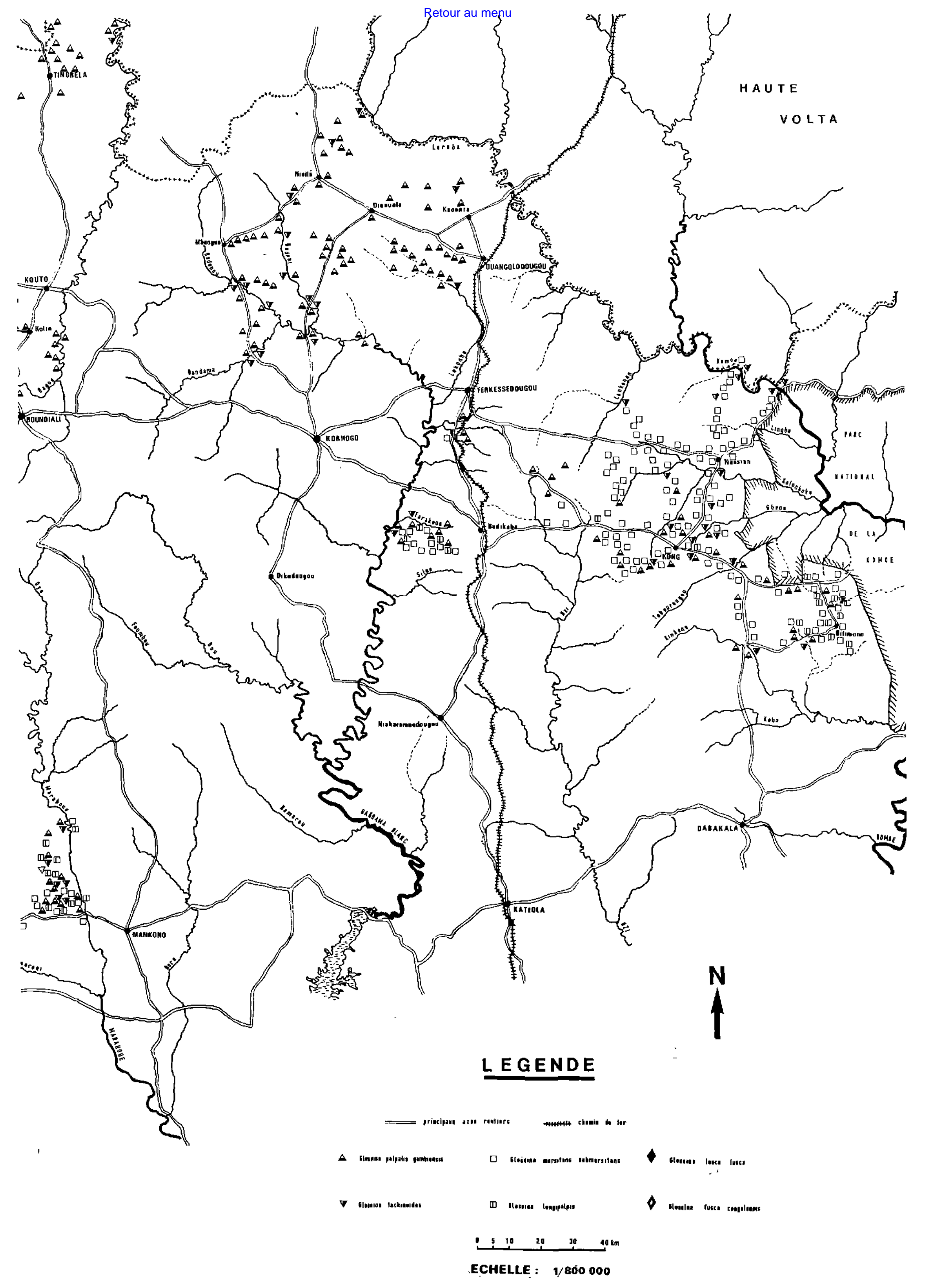


T.A. ${ }^{2}$

$\underbrace{\Delta}_{\Delta \Delta}$

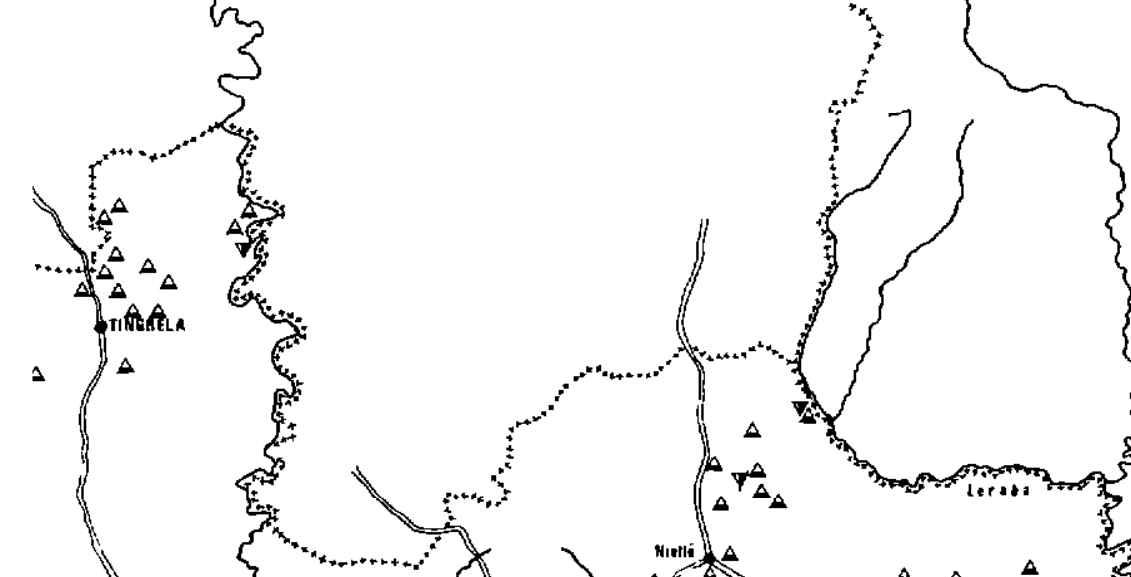

s 


\section{II.3. Techniques d'enquête}

Les enquêtes ont été réalisées à différentes périodes de l'année, tant en saison sèche qu'en saison des pluies.

A l'exception de l'enquête de 1971 (5) qui concerne le ranch de Sipilou et les régions de Man, Touba et Borotou durant laquelle les captures ont été effectuées manuellement par une équipe de captureurs, les missions furent réalisées à l'aide du piège biconique (4). Chaque matin, l'entomologiste et un aide ont placé une vingtaine de pièges dans des endroits écologique- ment propices aux glossines. Ils ont été relevés au cours de l'après-midi. Les captures ont donc eu lieu dans les mêmes conditions et pendant un laps de temps constant.

La densité réelle des glossines sur d'aussi grandes superficies est actuellement impossible à déterminer. Pour l'ensemble des enquêtes, mise à part celle de 1971, la seule approche possible consiste à exprimer le nombre de mouches capturées par rapport au moyen de capture, en l'occurrence le piège biconique, régulièrement utilisé. Les différentes espèces de glossines n'étant pas attirées de la même façon par celuici, 3 échelles de valeur ont été définies.

TABLEL N $\mathbb{N}^{\circ} \mathrm{I}$

\begin{tabular}{|c|c|c|c|c|}
\hline Echelle & Symbole & 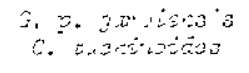 & G. m. submorsitane & G. Zongipatpis \\
\hline Très forte densité & ++++ & $>15 \mathrm{~g} / \mathrm{N} / \mathrm{j}$ & $>10 \mathrm{~g} / \mathrm{p} / \mathrm{J}$ & \multirow{2}{*}{$\geqslant 2 \mathrm{~g} / \mathrm{p} / \mathrm{j}$} \\
\hline Farte densité & +++ & 10 à $15 \mathrm{~g} / \mathrm{m} / \mathrm{J}$ & $6 \mathrm{a} 10 \mathrm{~g} / \mathrm{p} / \mathrm{j}$ & \\
\hline Moyenne densitê & ++ & $3 \mathrm{a} 10 \mathrm{~g} / \mathrm{p} / \mathrm{j}$ & $2 \mathrm{~d} \quad 6 \mathrm{~g} / \mathrm{p} / \mathrm{j}$ & \multirow{2}{*}{$\leqslant 2 g / p / j$} \\
\hline Faible clensité & + & $<3 \mathrm{~g} / \mathrm{p} / \mathrm{j}$ & $<2 g / p / j$ & \\
\hline
\end{tabular}

\section{4. Résultats}

Résultats par zone d'aménagement (cf. tableau I et carte de répartition)

Ranch de Sipilou et région de Man, Touba, Borotou

Toutes les galeries forestières bordant les cours d'eau permanents ou semi-permanents abritent Glossina palpalis gambiensis. Dans les parties boisées du ranch de Sipilou, ainsi qu'au nord de celui-ci, en bordure du Bafing, quelques individus du groupe fusca (sous-genre Austenina), appartenant à 2 espèces (G. fusca fusca et $G$. fusca congolensis) ont été capturés.

La présence de $G$. $m$. submorsitans a été mise en évidence près de Borotou, en bordure d'une forêt classée.

\section{Ranch de la Marahoué}

Quatre espèces de glossines sont présentes sur ce ranch: G. p. gambiensis et G. tachinoides, espèces ripicoles; G. m. submorsitans et $G$. longipalpis dans les savanes boisées. Les densités des 2 premières espèces sont faibles ou moyennes; celles des secondes sont moyennes, voire même fortes en ce qui concerne $G$. longipalpis.

\section{Porte d'entrée du bétail de Tienko}

Deux espèces y sont présentes en fortes densités, même en saison sèche : G. p. gambiensis qui se concentre dans les cordons ripicoles et G. $m$. submorsitans qui occupe les savanes de bas-fonds bien protégées.

\section{Porte d'entrée du bétail de Tingréla}

Cette zone renferme essentiellement G. $p$. gambiensis qui se localise aux marigots même complètement à sec, à galerie peu dense et parfois détruite par le feu. Trois exemplaires seulement de $G$. tachinoides ont été capturés sur la Bagoé.

Portes d'entrée du bétail de Niellé et Ouangolodougou

Seule G. p. gambiensis est présente dans le cours supérieur des marigots asséchés, là où un couvert végétal suffisant leur assure une certaine protection.

\section{Zone sylvo-pastorale du Lokpoho}

Deux espèces y sont présentes, G. $p$. gambiensis sur la majorité des cours d'eau permanents ou à 
TABL. $N^{\circ}$ IT.Captures de glossines dans les différentes zones de prospections.

\begin{tabular}{|c|c|c|c|c|}
\hline Zones de prospections & E s pẽ $c e s$ & $\begin{array}{c}\text { Nombre cic glossines } \\
\text { pax espèce }\end{array}$ & $\begin{array}{l}\text { Combre total de } \\
\text { dossines capturëes }\end{array}$ & $\begin{array}{c}\text { Densité moyenne } \\
\text { de glossines/p/jour }\end{array}$ \\
\hline $\begin{array}{l}\text { Ranch de Sipilou } \\
\text { Region de Man } \\
\text { Touba Barotou } \\
\text { (pour mémoire) } 1977\end{array}$ & $\begin{array}{l}\text { G. T. gombonsis } \\
\text { G. m. submonsizans } \\
\text { G. f. fusea } \\
\text { G. p. congoiensis }\end{array}$ & $\begin{array}{l}- \\
-\end{array}$ & $\begin{array}{l}- \\
- \\
-\end{array}$ & $\begin{array}{l}- \\
- \\
-\end{array}$ \\
\hline $\begin{array}{l}\text { Ranch de la Marahouê } \\
\text { (saison sèche 1977) }\end{array}$ & $\begin{array}{l}\text { G. p. grmbiensis } \\
\text { G. tachinoiles } \\
\text { G. m. suborsitans } \\
\text { C. Zongipalpis }\end{array}$ & $\begin{array}{l}64 \\
39 \\
31 \\
18\end{array}$ & 152 & 4 \\
\hline $\begin{array}{l}\text { Porte de Tienko } \\
\text { (saison sèche 1975) }\end{array}$ & $\begin{array}{l}\text { G. p. gamientsus } \\
\text { G. Eachinoides } \\
\text { G. m. subinorszans }\end{array}$ & $\begin{array}{r}97 \\
176 \\
13\end{array}$ & 286 & 5,7 \\
\hline $\begin{array}{l}\text { Porte de Tingrela } \\
\text { (saison sêche 1975) }\end{array}$ & $\begin{array}{l}\text { G. p. gambie.rsis } \\
\text { G. tachinoides }\end{array}$ & $\begin{array}{r}294 \\
3\end{array}$ & 297 & 5,0 \\
\hline $\begin{array}{l}\text { Porte de Niellé } \\
\text { (saison sêche 1975) }\end{array}$ & $\begin{array}{l}\text { a. p. ganbiensis } \\
\text { G. tachinotides }\end{array}$ & $\begin{array}{l}74 \\
27\end{array}$ & 101 & 1,3 \\
\hline $\begin{array}{l}\text { Porte de Ouangolodougou } \\
\text { (saison steche 1975) }\end{array}$ & $\begin{array}{l}\text { a. p. ambiensis } \\
\text { a. tachincioes }\end{array}$ & $\begin{array}{r}27 \\
1\end{array}$ & 28 & 1,2 \\
\hline $\begin{array}{l}\text { Zone sylvo-pastorale } \\
\text { du Lokpoho } \\
\text { (saison sêche 1976) }\end{array}$ & $\begin{array}{l}\text { G. p. gombiensis } \\
\text { t. tachinoides }\end{array}$ & $\begin{array}{r}1321 \\
222\end{array}$ & 1543 & 8,4 \\
\hline $\begin{array}{l}\text { Zone de la Palé } \\
\text { (salson sêche 1975) }\end{array}$ & $\begin{array}{l}\text { G. p. gambiensio } \\
\text { G. Lachinoides } \\
\text { G. th. oubmorsitane }\end{array}$ & $\begin{array}{r}568 \\
4 \\
2\end{array}$ & 574 & 3,4 \\
\hline $\begin{array}{l}\text { Zone de la Pale } \\
\text { (saison des pluies } 75 \text { ) }\end{array}$ & $\begin{array}{l}\text { G. p. gambiensis } \\
\text { G. tachinoides } \\
\text { G. in. sumorsitano }\end{array}$ & $\begin{array}{r}799 \\
31 \\
3\end{array}$ & 833 & 4,2 \\
\hline $\begin{array}{l}\text { Zone de la Pale } \\
\text { (saison sêche 1977) }\end{array}$ & $\begin{array}{l}\text { G. p. gombiensis } \\
\text { G. tachinoides } \\
\text { G. m. submcrsitans }\end{array}$ & $\begin{array}{l}182 \\
127 \\
467\end{array}$ & 776 & 6,3 \\
\hline $\begin{array}{l}\text { Ranch de Ferkessédougou } \\
\text { (Avril - Mai 1975) }\end{array}$ & $\begin{array}{l}\text { G. p. gambiensis } \\
\text { G. m. subnorsitinns } \\
\text { (pour mëmoire) }\end{array}$ & $\begin{array}{r}77 \\
-\end{array}$ & $\begin{array}{r}77 \\
-\end{array}$ & 4,5 \\
\hline $\begin{array}{l}\text { Ferme de la Sodepra } \\
\text { (saison sèche 1977) }\end{array}$ & $\begin{array}{l}\text { G. p. Gambiensis } \\
\text { G. tachinotdes } \\
\text { G. m. submorsitans } \\
\text { G. Zneipalpts }\end{array}$ & $\begin{array}{r}28 \\
36 \\
14 \\
5\end{array}$ & 83 & 2,9 \\
\hline $\begin{array}{l}\text { Zone de Kang } \\
\text { (saison sèche 1977) }\end{array}$ & $\begin{array}{l}\text { G. p. gandiensis } \\
\text { G. tachinoides } \\
\text { G. m. submorsitans } \\
\text { G. Longipatpis }\end{array}$ & $\begin{array}{r}116 \\
339 \\
1509 \\
22\end{array}$ & 1986 & 10,8 \\
\hline
\end{tabular}

sec et $G$. tachinoides, que l'on rencontre essentiellement sur les rivières à galerie relativement large, à canopée non jointive et dont le lit renferme au moins quelques grandes mares permanentes. La densité moyenne y est assez forte et des densités maximales de $170 \mathrm{~g} / \mathrm{p} / \mathrm{j}$ ont été relevées sur le cours inférieur du Badenou.

\section{Zone d'aménagement sylvo-pastoral de la Palé}

Trois enquêtes ont été réalisées dans cette zone, durant la saison sèche 1975, la saison des pluies 1975 et la saison sèche 1977 .

Trois espèces de glossines sont présentes. G. p. gambiensis occupe les galeries forestières de l'ensemble du réseau hydrographique ; G. tachinoides n'est capturée que sur des rivières plus importantes (Palé, Tyoro, Bagoé). Ces 2 espèces présentent des densités faibles ou moyennes.

G. $m$. submorsitans est présente dans les savanes arborées et les forêts claires de l'ouest et du centre du périmètre aménagé. Ses densités sont fortes ou très fortes avec un point de capture record de $129 \mathrm{~g} / \mathrm{p} / \mathrm{j}$.

\section{Ranch d'embouche au sud de Ferkéssédougou}

L'enquête réalisée en avril et mai 1976 a révélé, le long des 2 galeries forestières denses mais peu larges, la présence, en faible densité, 
de G. p. gambiensis. G. m. submorsitans, alors absente, est apparue depuis l'introduction du bétail mais les densités restent faibles.

\section{Ferme semencière de la SODEPRA}

Quatre espèces y sont présentes : G. p. gambiensis et $G$. tachinoides dans les galeries forestières ; G. m. submorsitans et $G$. longipalpis dans les îlots de forêt claire et près des galeries forestières. Les densités sont faibles ou moyennes.

\section{Zone agro-pastorale de Kong}

G. p. gambiensis est présente en faible ou moyenne densité sur la quasi-totalité du réseau hydrographique.

G. tachinoides fréquente les galeries forestières larges à canopée non jointive des cours d'eau importants. Ses densités sont moyennes.

G. m. submorsitans est présente dans les savanes boisées et les forêts claires ainsi que près des galeries forestières sur l'ensemble de la zone à l'exception de l'extrême ouest. De très fortes et parfois exceptionnellement fortes densités se rencontrent dans les régions les plus giboyeuses. Ces densités diminuent au fur et à mesure que l'on se dirige vers le centre et l'ouest de la zone.

G. longipalpis est capturée dans les forêts claires et les galeries forestières de la partie sudest. Les densités semblent fortes.

\section{Résultats par espèce}

Six espèces appartenant aux 3 groupes de glossines ont été capturées au cours de ces enquêtes.

Groupe palpalis (sous genre Nemorhina)

a) G. p. gambiensis

Cette glossine est présente dans toutes les zones d'étude. Elle est même parfois la seule existante. On la rencontre dans les galeries forestières des marigots, que ceux-ci s'assèchent complètement ou qu'ils conservent des mares permanentes pendant toute la saison sèche. Elle est également présente, associée à G. tachinoides, dans les galeries forestières des cours d'eau importants. Ses hôtes nourriciers préférentiels étant les reptiles puis les mammifères et l'homme, elle trouve partout les conditions favorables à son alimentation. La présence constante de cette espèce est importante à souligner, car, bon vecteur des trypanosomes animaux, elle consti- tue le vecteur principal de la trypanosomiase humaine en Afrique de l'Ouest.

\section{b) G. tachinoides}

Moins largement répandue que l'espèce précédente, elle peut présenter des densités importantes quand les conditions écologiques de son maintien sont réunies : galerie forestière large et assez dense, à canopée ouverte et présence d'eau, au moins sous forme de mares, toute l'année. Très ubiquiste quant à son alimentation, sa densité n'est pas liée à la présence d'un hôte nourricier préférentiel, qui n'influe pas non plus sur son aire de répartition.

Groupe morsitans (sous genre Glossina s. str.)

a) G. m. submorsitans

Mouche de savane par excellence, elle est présente dans les secteurs prospectés qui comportent des zones de savanes boisées plus ou moins denses à Isoberlinia doka. Sa présence semble essentiellement liée à celle du gibier et tout particulièrement du phacochère. On ne la trouve que dans les zones d'étude qui abritent encore une faune relativement importante de grands mammifères. Elle peut alors présenter de très fortes densités.

\section{b) G. longipalpis}

Egalement mouche de savane, mais à hygrophilie et ombrophilie plus prononcées, on la rencontre plus au sud que l'espèce précédente; elle devient progressivement dominante au fur et à mesure que le déficit hydrique cumulé diminue et que la végétation arborée devient plus dense. Elle semble également liée à la présence des grands mammifères qui constituent ses hôtes nourriciers préférentiels.

\section{Groupe fusca (sous genre Austenina)}

Ce groupe est celui des mouches de forêt dense et humide. Deux espèces sont présentes sur le ranch de Sipilou situé en secteur guinéen.

\section{DISCUSSION}

\section{(cf. carte de répartition)}

\section{III.1. Réalisation de la carte}

Selon la superficie prospectée lors de chaque enquête, chacune des cartes ponctuelles a été 
établie à une échelle appropriée allant $d u$ $1 / 20000^{\mathrm{e}}$ au $1 / 200000^{\mathrm{e}}$. La carte de synthèse a été réalisée à l'échelle $1 / 800000^{\mathrm{e}}$.

Les espèces de glossines y sont représentées par les signes conventionnels internationaux. Chaque symbole signifie qu'une mouche au moins de cette espèce était présente dans le piège situé à cet endroit. La densité n'est pas représentée.

La toponymie est celle de la carte d'Afrique de l'Ouest au $1 / 200000^{\mathrm{e}}$ dressée par l'I. G. N. (10).

\section{III.2. Synthèse des enquêtes}

Ces enquêtes mettent en évidence les différences qui existent dans la répartition et la densité des glossines, entre des régions parfois peu éloignées les unes des autres. A l'intérieur même d'une zone prospectée, les répartitions ne sont pas uniformes.

Ces disparités tiennent aux conditions écologiques. En effet, chaque espèce de glossines possède des exigences plus ou moins strictes en ce qui concerne le climat, la végétation et la présence d'hôtes nourriciers préférentiels.

Certaines sont ubiquistes, telles G.p. gambiensis et G. tachinoides que l'on rencontre sur la presque totalité du nord de la Côte-d'Ivoire. Ces mouches tributaires des galeries forestières, $y$ trouvent des conditions climatiques (température, humidité, ombre, protection contre le vent) à peu près identiques quelle que soit la zone phytoclimatique envisagée. Il a cependant été noté, plus haut, la préférence de G. tachinoides pour une certaine forme de végétation ripicole, large et non jointive qui accompagne des cours d'eau plus ou moins permanents. Pour ces 2 espèces, la présence d'un hôte nourricier préférentiel ne se pose pas, les galeries forestières restant le dernier refuge de la faune résiduelle (reptiles, rongeurs, petits ruminants sauvages) lorsque celle-ci a disparu des savanes environnantes.

G. m. submoristans est une souche de savane arbustive ou arborée soudanienne et subsoudanienne. L'élément écologique primordial qui détermine sa présence semble être l'existence de gibier et tout particulièrement du phacochère, son hôte nourricier préféré. Ainsi les zones de la Palé et de Kong abritent cette espèce de glossines sur la plus grande partie de leur surface.
Cependant dans l'un et l'autre cas, une petite région (à l'est pour la Palé, à l'ouest pour Kong) est indemne de $G$. $m$. submorsitans. La population humaine y est plus dense que dans le reste des zones prospectées, ce qui entraîne un déboisement parfois intensif pour la mise en culture des terres et une disparition du gibier.

Un exemple inverse est donné par le ranch d'embouche au sud de Ferkéssédougou. Tant qu'il s'agissait d'une plantation de Kénaf, et avant l'introduction de bétail, aucune $G . m$. submorsitans n'y a été capturée à l'occasion d'enquêtes antérieures. Depuis que les champs ont été transformés en pâturages, que des bovins y sont embouchés, et que la chasse y est interdite, le gibier semble avoir un peu augmenté. G. $m$. submorsitans, certainement arrivée par la route de bétail qui longe le ranch, s'y maintient aujourd'hui.

Enfin, dans toutes les régions où cette espèce a été capturée, il a été possible d'observer des phacochères.

G. longipalpis présente les caractères identiques à ceux de G. m. submorsitans mais est plus exigeante en ce qui concerne l'environnement climatique et végétal. On ne la rencontre qu'en zone guinéenne ou subsoudanienne, là où des forêts claires ou des forêts denses sèches lui permettent de trouver refuge pendant la saison sèche. Bien que son écologie soit encore assez mal connue, elle semble également liée à la présence du gibier.

Dans les sites où elle est capturée, sa densité est en général assez forte.

Son aire de répartition chevauche celle de G. $m$. submorsitans, mais alors qu'en zone subsoudanienne elle est minoritaire (zone de Kong, ferme semencière de la SODEPRA) elle devient dominante dans les savanes guinéennes (Ranch de la Marahoué).

Quelques glossines du groupe fusca ont été capturées uniquement près de Man, en limite de la forêt guinéenne semie décidue. Leur écologie est encore très mal connue.

\section{III.3. Comparaison avec les cartes antérieures}

Malgré leurs imprécisions, les cartes citées plus haut (cf. $\S \mathrm{I}$ ) ont rendu de grands services dans le passé et il est très intéressant d'y noter l'évolution des aires de répartition des différentes espèces de 1948 à 1977. 


\section{Sous genre Nemorhina}

- Glossina palpalis gambiensis. La répartition uniforme sur l'ensemble du nord de la Côted'Ivoire ne s'est pas modifiée depuis 1948.

- Glossina tachinoides. En 1948, la limite sud de répartition de cette espèce était signalée au nord-est de Boundiali, au sud de Korhogo et au nord-est de Dabakala. En 1961, l'aire de répartition s'était étendue vers le sud et des captures étaient répertoriées dans les degrés carrés d'Odiénné et de Katiola. En 1977, 39 spécimens de cette espèce ont été capturés sur le Ranch de la Marahoué, c'est-à-dire dans le degré carré de Mankono (19). Il s'agit là d'une nouvelle localisation.

\section{Sous genre Glossina s. str.}

- Glossina morsitans submorsitans. En 1949, la limite ouest de l'aire de répartition de cette espèce était signalée entre Korhogo et Ferkéssédougou et la limite sud, au sud de Dabakala et au nord de Bondoukou. En 1961, elle était repoussée plus à l'ouest et au sud-ouest, aux degrés carrés de Niellé, Katiola, Mankono, Séguéla et Touba. En 1975 et 1977, des glossines de cette espéce ont été capturées, encore plus à l'ouest, dans les degrés carrés de Tienko et Boundiali ( 6 et 17). Il s'agit de nouveaux lieux de capture de l'espèce. En revanche, elle semble avoir disparu des régions proches de Korhogo et Ferkéssédougou, probablement en raison des modifications du milieu naturel (cf. III.2).

Comme pour $G$. tachinoides, l'aire de répartition de G. m. submorsitans s'avère être plus étendue vers l'ouest et le sud que ne l'indiquaient les cartes antérieures, peut-être du fait de son installation dans des régions autrefois indemnes.

- Glossina longipalpis. Situation stable entre 1949 et 1961. Au cours des enquêtes réalisées récemment, aucun spécimen de cette espèce n'a été capturé dans les degrés carrés de Korhogo, Boundiali et Touba où sa présence était signalée par les cartes antérieures. S'agit-il d'une régression vers le sud de l'aire de répartition? Il est impossible de conclure, les captures ne couvrant pas l'ensemble des régions pré-citées.

\section{Sous genre Austenina}

Les quelques captures d'individus appartenant à ce groupe, réalisées en 1971 (5), se situent à la limite de leur aire de répartition. La situation semble stable.
Glossina medicorum a été signalée sur la Komoë, à la frontière entre la Côte-d'Ivoire et la Haute-Volta.

Elle a en effet été capturée, en 1976-1977 par les entomologistes du projet OMS/ TRYPANO de Bobo-Dioulasso. Cependant elle n'a pas été retrouvée lors de l'enquête que nous avons effectuée en 1977.

\section{CONCLUSION}

Les enquêtes entomologiques effectuées en différents points du nord de la Côte-d'Ivoire de 1971 à 1977 ont permis de mettre en évidence la présence de 6 espèces de glossines appartenant aux 3 groupes classiquement reconnus :

\section{Groupe palpalis (S. g. Nemorhina)}

Glossina palpalis gambiensis Vanderplank, 1949.

Glossina tachinoides Westwood, 1850.

Groupe morsitans (S. g. Glossina s. str.)

Glossina morsitans submorsitans Newstead, 1910.

Glossina longipalpis Wiedemann, 1830.

Groupe fusca (S. g. Austenina)

Glossina fusca fusca Walker, 1849.

Glossina fusca congolensis Newstead et Evans, 1921.

La comparaison avec les cartes précédentes montre une extension des aires de répartition connues de $G$. tachinoides et $G$. m. submorsitans vers l'ouest et le sud.

Ces enquêtes ponctuelles ne peuvent donner qu'une idée fragmentaire des répartitions des différentes espèces et de leurs densités dans le nord de la Côte-d'Ivoire.

L'étude des facteurs écologiques conditionnant la présence des glossines montre l'importance que jouent le climat, la végétation, la présence du gibier et du bétail et les densités de population humaine.

Il est donc fort souhaitable qu'une prospection entomologique systématique de tout le nord du pays précise la répartition actuelle exacte des 
espèces, leurs densités et l'évolution de leurs aires de répartition en fonction des variations des conditions écologiques.

Cette enquête qui sera réalisée au cours des 3 prochaines années, complètera les données éparses sur ce problème et pérmettra de concevoir de façon globale, les moyens à mettre en cuvre pour organiser un aménagement humain, pastoral et agricole de la zone des savanes en Côte-d'Tvoire.

\section{SUMMARY}

\section{Tsetse fly distribution in the North of Ivory Coast}

Six species were recorded G. p. gambiensis, G. tachinoides G. m. submorsitans, $G$. longipalpis, $G$. fusca fusca, $G$. fusca congolensis.

Although these point surveys only give a fragmentary view of the species distribution and density, they allow to locate foci not recorded on previous distribution maps, especially $G$. tachinoides and $G$. m. submorsitans foci.

\section{RESUMEN}

\section{Contribución al estudio de la repartición}

de las glosinas en el norte de la Costa de Marfil

Se empadronaron seis especies: G. p. gambiensis, G. tachinoides, G. $m$. submorsitans, G. longipalpis, G. fusca fusca, G. fusca congolensis.

Aunque estas encuestas puntuales no den más que una idea fragmentaria de las reparticiones de las especies y de su densidad, permitieron notar la existencia de focos no señalados en las mapas de repartición anteriores, particularmente en Io concerniendo G. tachinoides y G. m. submorsitans.

\section{BIBLIOGRAPHIE}

1. AVENARD (J. M.) et collab. Le milieu naturel de la Côte-d'Ivorre. I. Mémoire, Il. Cartes, Paris, O. R. S. T. O. M., 1971.

2. CHALlIER (A.). Rapport: Projet de lutte antiglossines. Kouto, Côte-d'Ivoire.

3. CHALLIER (A.), LAVEISSIÈRE (C.). La répartition des glossines en Haute-Volta. Notıce explicative $n^{0}$ 69. Carte à 1/2000 000, Paris, O.R.S.T.OM., 1977.

4. CHALLIER (A.), LAVEISSIËRE (C.). Un noLveau piège pour la capture des glossines (Glossina: Diptera, Muscidae). Description et essass sur le terrain. Cah. O. R. S. T. O. M., sér. Ent. méd. Parasitol., 1973,11 (4) : $251-262$.

5. CLAIR (M.) Etude entomologique in: Etude de factibilité des ranches d'Abokouamékro et de Sipilou en République de Côte-d'Yvoire. Massons-Alfort, I. E. M. V. T., 1972.

6. CUISANCE (D.). Enquête sur les glossines dans la zone de la Palé et au niveau des portes d'entrée du bétatl dans le nord de la Côte-d'Ivoire. MaisonsAlfort, I. E. M. V. T., 1975, 105 p. (Travail exécuté à la demande et pour le compte du B. N. E. T. D., Abidjan).

7. FINELLE (P). Protection santaire du cheptel bovin dans le nord de la côte-d'Ivoire. MaisonsAlfort, 1. E. M. V. T., 1971, 53 p.

8. FORD (J.). Distribution of the vectors of african pathogenics trypanosomes. Bull. Org. mond. Santé, $1963,28,653-659$.

9. FORD (J.), KATONDO (K, M.). The distribution of tsetse flies (Glossina) in Africa. Nairobi, OUA/ STRC, 1973.

10. I. G. N. Carte de l'Afrique Occidentale au 1/200000. Paris, Institut Géographique National, 1975.

11. ITARD (J.), FINELLE (P.). Rapport de mission préparatoire au projet d'étude de l'infestation glossinaire en Côte-d'Ivoire. Rapport P. N. U. D./ R. A. F./76/020, février 1977.

12. POLITZAR $\left(\mathrm{H}_{1}\right)$. Enquête entomologıque sur les glossines dans le futur ranch d'embouche situé à $22 \mathrm{~km}$ au sud de Ferkessédougou. Rapport interne non publié. Mai 1976.

13. POTTS (W. H.). Distribution of tsetse species in Africa. Sheet I : West Africa. Compiled and drawn by Directorate of Colonial Suveys ; informations collected by Potts. 1953.

14. RICKENBACH (A.). Carte de répartition des glossines en Afrique Occidentale d'expression française. O.R.S.T.O.M., Bobo-Dioulasso, Centre Muraz, 1961.

15. ROUBAUD (E.). Les mouches tsétsé en Afrique Occidentale Française, Nos connaissances actuelles sur leur histoire et leur rôle pathogene. Bull. Com. Etud. hist. Sci. A. O. F., 1920, 3, 257-300.

16. TAZE (Y.). Enquête sur les glossines dans la zone sylvo-pastorale du Lokpoho, Maisons-Alfort, I. E. M. V. T., 1976, 30 p. (Travail exécuté à la demande et pour le compte du B.N.E.T.D., Abidjan).

17. TAZE (Y.). Enquête sur les glossines dans le cadre du suivi technique de la zone sylvo-pastorale de la Palé. Maisons-Alfort, I. E. M. V. T., 1977, 14 p. (Travail exécuté à la demande et pour le compte du B. N. E. T. D., Abidjan).

18. TAZE (Y..) Enquête sur les glossines dans la zone agro-pastorale de Kong. Maisons-Alfort. I. E. M. V. T., 1977, 19 p. (Travail exécuté à la demande et pour le compte du B.N.E.T. D., Abidjan).

19. TAZE (Y.). Enquête sur les glossines dans le ranch de la Marahoué. Maisons-Alfort, I. E. M. V. T., 1977, 15 p. (Travaíl exécuté à la demande et pour le compte du FED, Abidjan).

20. TAZE (Y.). Enquête sur les glossines sur la ferme semencière de la S. O.D. E. P. R. A. MaisonsAlfort, I. E. M. V. T., 1977, 6 p.

21. TAZE (Y.), CUISANCE (D.), POLITZAR (H.). Les glossines dans le nord de la Côte-d'Tvoire: contrainte sur le développement de l'élevage. In : Actes du Colloque de Bouaké sur l'élevage en zone tropicale humide. Maisons-Alfort, I. E. M. V. T., 1978. (sous presse).

22. VILAIN (P.). De la répartition géographique des glossines en Afrique Occidentale Française. Bull. méd. A. O. F., 1948, 5, 107-116.

23. W.I.P. Etude des possibilités de création de ranches d'élevage en Côte-d'Ivoire. Choix des zones d'élevage. Tome III ; Entomologie, Parasitologie et Zootechnie, janvier 1971. 